\title{
Modulation of the Gloeobacter violaceus Ion Channel by Fentanyl: A Molecular Dynamics Study
}

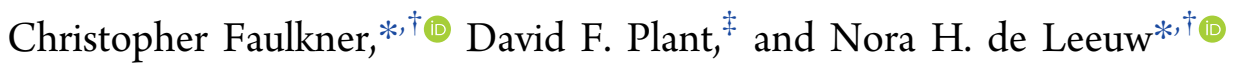 \\ ${ }^{\dagger}$ School of Chemistry, Cardiff University, Main Building, Park Place, Cardiff CF10 3AT, United Kingdom \\ ${ }^{\ddagger}$ Atomic Weapons Establishment (AWE), Aldermaston, Reading RG7 4PR, United Kingdom
}

Supporting Information

ABSTRACT: Fentanyl is an opioid analgesic, which is routinely used in general surgery to suppress the sensation of pain and as the analgesic component in the induction and maintenance of anesthesia. Fentanyl is also used as the main component to induce anesthesia and as a potentiator to the general anesthetic propofol. The mechanism by which fentanyl induces its anesthetic action is still unclear, and we have therefore employed fully atomistic molecular dynamics simulations to probe this process by simulating the interactions of fentanyl with the Gloeobacter violaceus ligand-gated ion channel (GLIC). In this paper, we identify multiple extracellular fentanyl binding sites, which are different from the transmembrane general anesthetic binding sites observed for propofol and other general anesthetics. Our simulations identify a novel fentanyl binding site within the GLIC that results in conformational changes that inhibit conduction through the channel.

$\mathrm{T}^{\mathrm{k}}$ he identification of opioid molecules, such as morphine and fentanyl, which cause desensitization to painful stimuli by acting upon G-protein-coupled receptors (GPCRs), has allowed great advances in modern medicine and invasive surgery. Other uses have also been identified for opioids such as fentanyl, i.e., in the potentiation of the general anesthetic propofol and as the main anesthetic component. ${ }^{1,2}$ However, the mechanism of the anesthetic action of opioids remains unclear. Here, we have investigated target ion channels, specifically the Cys-loop family of pentameric ligand-gated ion channels (pLGICs). These proteins are sensitive to neurotransmitters from the presynaptic axon terminal and are hence major drug targets. ${ }^{3}$ Anesthetics are known to modulate both cation- and anion-permeable channels, such as the $\gamma$ Aminobutyric acid type A (GABAA) and nicotinic acetylcholine receptors (nAChRs), ${ }^{4,5}$ but high-resolution structures of eukaryotic receptors have proved to be challenging to obtain. Crystal structures of the bacterial homologue (GLIC) have been obtained at reasonably high resolutions (2.4-4 $\AA){ }^{6}$ which allows researchers the opportunity to study the modulation of pLGIC at the atomic level. This family of ion channels was chosen on the basis of evidence from other studies that show an "anesthetic binding pocket" that general anesthetics have been shown to occupy. ${ }^{7,8}$ The analgesic/ anesthetic drug ketamine has also been shown to bind to the
GLIC structure $^{9}$ in a different, extracellular binding site compared to that of general anesthetics.

Figure 1 shows the structure of fentanyl and the transmembrane domain (TMD) of the GLIC. The TMD consists of

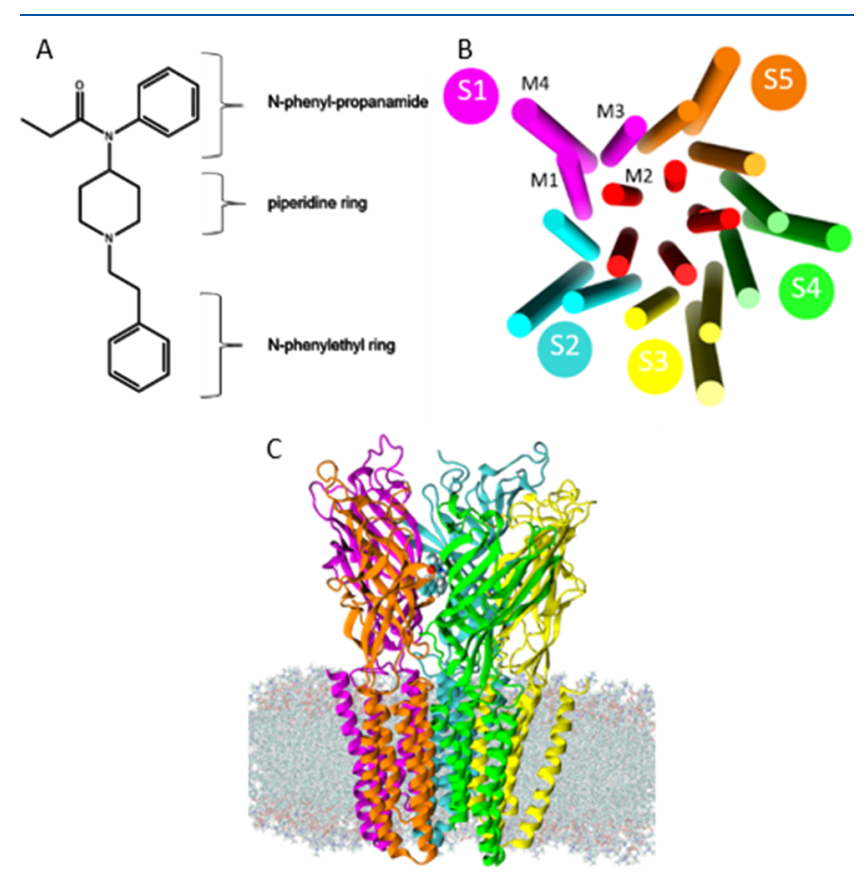

Figure 1. (A) Chemical structure of fentanyl. (B) Graphical representation of the transmembrane $\alpha$-helical domains showing the five symmetric subunits and the ion-conducting M2 helices (red). (C) GLIC structure in the membrane environment with fentanyl (spheres) in its intersubunit site.

four $\alpha$-helices that span the entirety of the cell membrane (M1-M4) in which the GLIC is imbedded. The M2 $\alpha$-helices are oriented toward the center of the pore that forms the ionconducting, fully hydrated channel in the open state. The evidence of anesthetics interacting with and modulating these channels ${ }^{10-12}$ provides an excellent starting point for the exploration of the anesthetic properties of fentanyl and how it can potentiate other general anesthetics.

Received: September 27, 2019

Revised: November 4, 2019

Published: November 13, 2019 
The GLIC open state structure at atomic resolution $(2.4 \AA)$ published by Sauguet et al. ${ }^{6}$ and the previous simulation studies of anesthetics interacting with ion channels ${ }^{13-15}$ provide us with the opportunity to compare the effect of fentanyl on a GLIC structure with the binding and modulation of the channel by general anesthetics. We have employed molecular dynamics (MD) simulations and efficient end state free energy calculations to probe the interactions between fentanyl and the GLIC. Our simulations reveal that fentanyl occupies multiple extracellular binding sites similar to those observed for ketamine, ${ }^{9}$ which lead to conformational changes within the M2 helix domain, causing pore closure and dehydration resulting in a nonconductive state.

We initially performed three separate 500 ns MD simulations on the pure GLIC structure inserted into a 1,2dioleoyl-sn-glycero-3-phosphocholine (DOPC) lipid bilayer ${ }^{6}$ as a reference, followed by three 500 ns simulations in which four fentanyl molecules were added to the simulation box in each system, where the equilibrated pure GLIC system before its production run was used as the starting structure. From the pure GLIC simulation, root-mean-square deviations (RMSDs) were calculated to assess whether the protein structure was stable within the membrane (Tables S1 and S2). Several binding sites were identified, and molecular mechanics Poisson-Boltzmann surface area (MMPBSA) ${ }^{16}$ calculations were performed to assess the strength of binding at each site. Figure 2 shows the residues in the fentanyl binding site, which

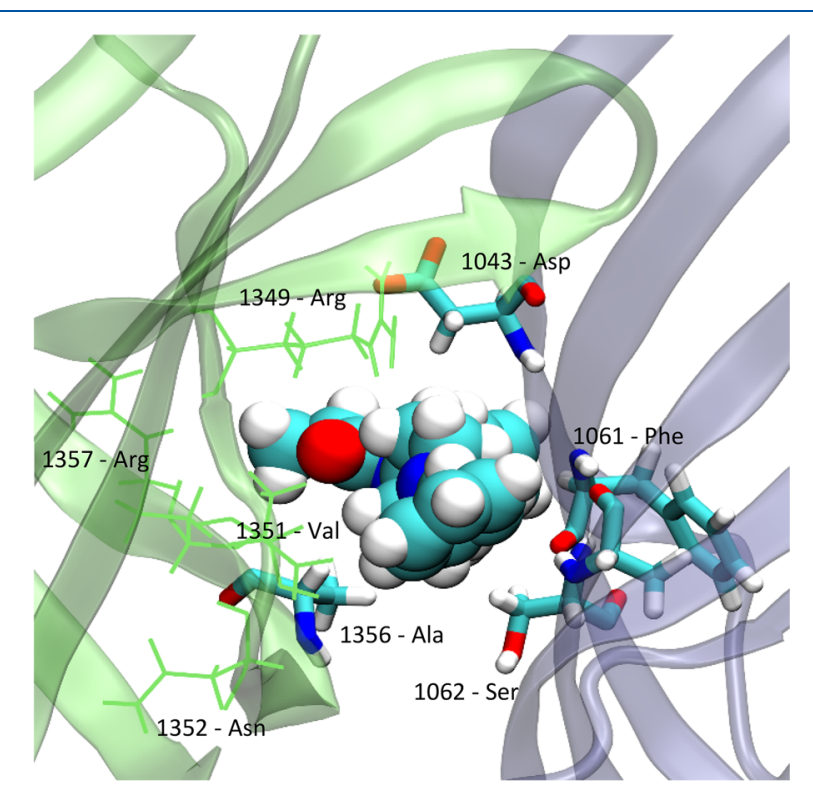

Figure 2. Detailed view of fentanyl binding in its intersubunit extracellular site. Fentanyl is shown in van der Waals sphere representation, with the strongest binding residues shown in licorice representation and additional residues shown in stick representation.

has a calculated binding free energy of $-27.35 \pm 0.06 \mathrm{kcal} / \mathrm{mol}$ (binding free energies and residues of other sites are listed in Tables S3-S8). Binding contributions from the ligand and the binding site residues are listed in Table S9.

The fentanyl molecule showing the strongest binding energy initially interacts with the hairpin loop located at the top of the extracellular domain for $\sim 20 \mathrm{~ns}$ before diffusing into the channel, where it then enters the binding pocket (Table 1). This binding pocket is situated between two subunits in the extracellular domain above the lipid headgroup region.
Table 1. Fentanyl Binding Sites in Order of Binding Free Energy

\begin{tabular}{ccc}
$\begin{array}{c}\text { extracellular binding } \\
\text { site }\end{array}$ & $\begin{array}{c}\text { residence time } \\
(\mathrm{ns})\end{array}$ & $\begin{array}{c}\text { binding free energy } \\
(\mathrm{kcal} / \mathrm{mol})\end{array}$ \\
1 & 450 & $-27.35 \pm 0.06$ \\
2 & 88.3 & $-18.88 \pm 0.18$ \\
3 & 78.1 & $-12.29 \pm 0.17$ \\
4 & 63.4 & $-12.00 \pm 0.20$ \\
5 & 57.9 & $-10.73 \pm 0.15$ \\
6 & 39.3 & $-8.29 \pm 0.11$ \\
7 & 9.7 & $-6.18 \pm 0.22$ \\
\hline
\end{tabular}

Fentanyl initiates binding at $\sim 40 \mathrm{~ns}$ in one simulation and at $\sim 50 \mathrm{~ns}$ in another, and they both remain within the binding site for the rest of the simulation time, totaling 460 and $450 \mathrm{~ns}$ of binding time, respectively, with little structural deviation from the binding conformation (RMSD $=1.7 \pm 0.8 \AA$ ), indicating that this is a very stable binding site. Secondary structure calculations show that there is no appreciable change in the secondary structure of the binding pocket (Figure S2) before or after fentanyl binding. Root-mean-square fluctuation calculations (Figure S8) for the two subunits that form the binding site show that fentanyl causes an increased fluctuation in S5 but stabilizes loop C, which forms the top of the binding site. At the other binding sites, fentanyl dissociated and diffused into the membrane domain where it remained for the rest of the simulations.

To assess the effects of binding on the function of the GLIC, we computed the change in the number of pore water molecules, using an in house python script utilizing the MDAnalysis python toolkit. ${ }^{17}$ The definition of pore water molecules is given in the section 1 of the Supporting Information. This methodology has previously been used to analyze hydrophobic gate formation in other channel proteins. ${ }^{18}$ Our analysis of the pure system confirmed that the channel was in the open state (Figure 3 ).

To identify visually the formation of the hydrophobic gate, $\mathrm{VMD}^{19}$ was used to identify the gating residues. From this information, we were able to identify residues 233-Ile and 240Ile as the residues forming the hydrophobic gate and causing dehydration of the M2 pore, which is consistent with the GLIC

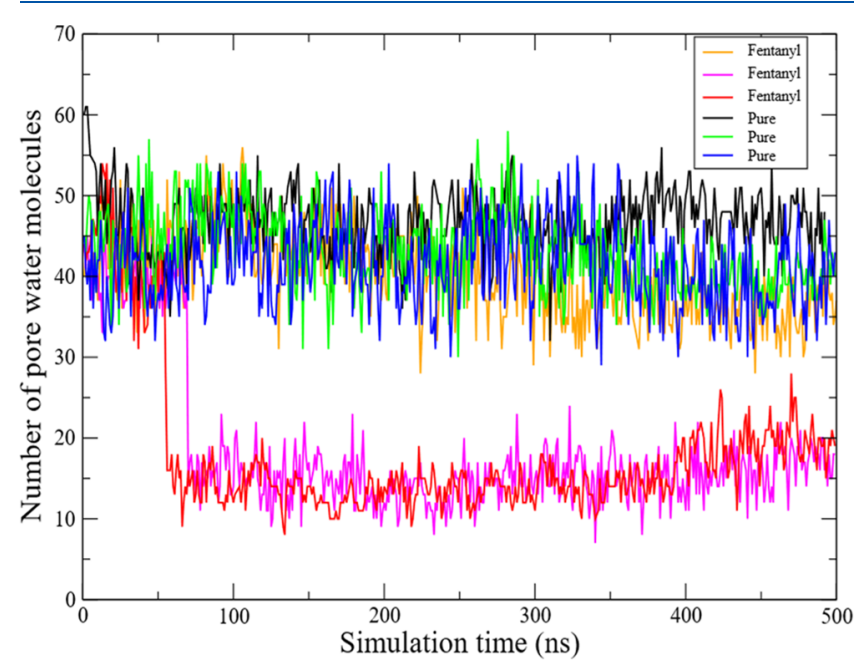

Figure 3. Plot highlighting the change in the number of pore water molecules within the M2 helix channel pore when fentanyl is bound. 
hydrophobic gating residues identified in other studies. ${ }^{7,20,21}$ This gating mechanism is similar to that for acetylcholine receptors that rely on M2 helix rotations to control ion conduction. $^{22}$

Our analysis clearly shows the formation of a hydrophobic gate within the pore that is consistent with that observed for general anesthetics. In addition, the M2 pore radius of the pure simulation is less contracted than that of the fentanyl system, instead resembling the open state of the crystal structure (Figure 4). As this change of state observed in the fentanyl

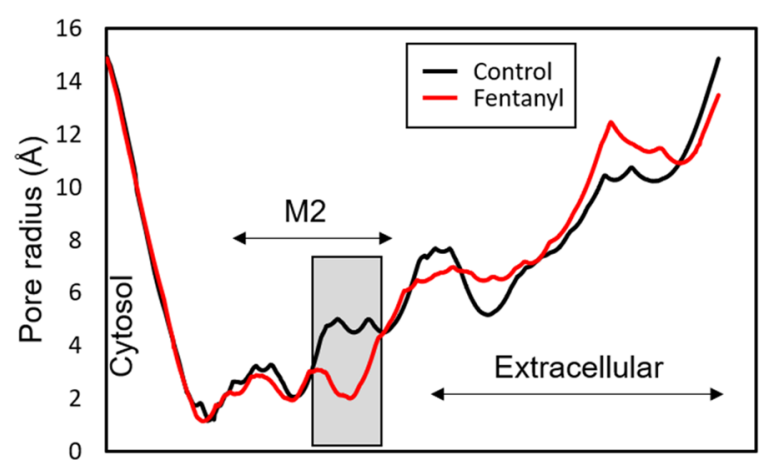

Figure 4. M2 pore radius calculations for the pure/control and fentanyl simulations averaged over the trajectories. The gray box represents the hydrophobic gate region. Calculations performed using HOLE. ${ }^{23}$

simulation is not observed within our control simulation, we suggest that hydrophobic gate formation is caused by the binding of the fentanyl molecule at $\sim 40 \mathrm{~ns}$.

To determine if the formation of the hydrophobic gate inhibited ion conduction through the channel, we have performed several applied electric field simulations (details given in section 1 of the Supporting Information). Ion conduction was observed in the pure GLIC system after $\sim 60$ $\mathrm{ns}$ at a transmembrane voltage of $270 \mathrm{mV}$, but no conduction was observed in the fentanyl-bound system, in which ions remained above the hydrophobic gate residues (Figure S10). Fentanyl remained bound during the course of these simulations.

The conformational change within the TMD associated with anesthetic binding is an $\sim 10^{\circ}$ tilt in the upper region of the M2 helix, causing an "iris-like" contraction., 20,24 To analyze the effect of fentanyl binding on the geometry of the M2 helix, we employed the TRAJELIX ${ }^{25}$ module as incorporated in the Simulaid analysis program, ${ }^{26}$ and the Bendix ${ }^{27}$ plugin for VMD. We used this methodology to calculate the global helix $x, y$, and $z$ tilt angles; the turn angle per residue; the local helix tilt; the helix rotation; and the angle of curvature for each M2 transmembrane helix over the course of the $500 \mathrm{~ns}$ trajectories. Full results of this analysis can be found in the Supporting Information. Figure 5 shows the helix rotation for one of the five M2 helices, as they were all relatively similar (see also Figures S3-S7). We consistently observed a rotation of $10^{\circ}$ with a small degree of local helix tilting, which suggests a method of pore contraction different from that observed in binding general anesthetics. ${ }^{7,20,24}$ From the curvature analysis, we observe that the general trend for the pure system is perturbed by fentanyl binding. The most noticeable increase in curvature is observed in the first 10 residues of the helix, which include the hydrophobic gate region.

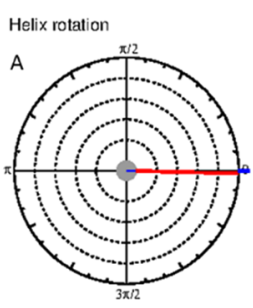

D

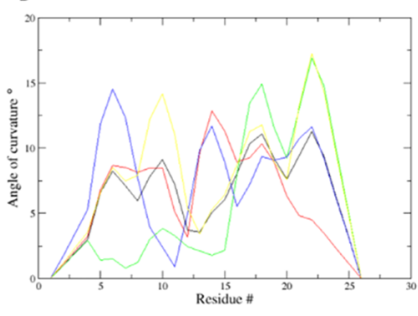

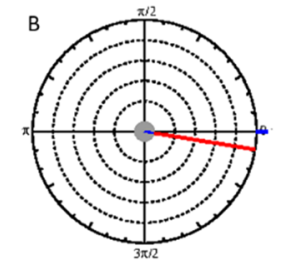

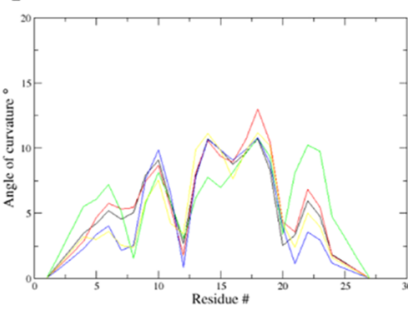

Figure 5. (A) Dial plot indicating the helix rotation of the S3M2 helix in the pure GLIC system. (B) Dial plot indicating the helix rotation of the S3M2 helix in the fentanyl-bound system. (C) Overlapped structures of the M2 TMD, with green indicating an open channel (fentanyl not bound) and red indicating a closed channel (fentanyl bound), with Ile gating residues shown in stick representation (rendered with UCSF Chimera ${ }^{28}$ ). (D and E) Helix curvature plots for fentanyl and pure simulations, respectively, where each line represents one subunit.

Although a full signaling pathway or mechanism of action for the action of neurotransmitters and drugs on pLGICs remains unclear, several studies have proposed conformational changes in certain regions of the channels. Salt bridge perturbation has been shown to be significant in the gating process for nicotinic and GABA receptors, as well as the GLIC. ${ }^{29,30}$ In our fentanylbound simulations, we have computed the distance between the center of mass (COM) of the 32-Asp and 192-Arg salt bridge (Figure S9), which was shown to break after fentanyl binding. Loop $\mathrm{C}$ was also stabilized in our simulations (Figure $\mathrm{S} 8$ ), which is also seen in various pLGIC ligand studies where loop $\mathrm{C}$ is stabilized in structurally different conformations. ${ }^{31}$

From this analysis, we can hypothesize that the difference in helix conformational change is due to the extracellular binding site, which has no direct interaction with the helices themselves. In contrast, the anesthetic binding site, which is either between TMD subunits or within the four helices of the subunits themselves, ${ }^{32-34}$ does directly interact with the M2 pore helices, possibly causing the larger degree of tilting that has been observed previously. We should note here that a 2.99 $\AA$ crystal structure has been obtained, which shows the anesthetic/analgesic drug ketamine binding to a GLIC structure with binding sites situated between extracellular subunits, ${ }^{9}$ which is consistent with our observations for fentanyl. Fentanyl remains deeper in the intersubunit site compared to ketamine, most likely due to the size of the fentanyl molecule. However, during our simulations, fentanyl was seen to interact with 154-Asp, which is the residue that contributes to the stabilization of ketamine. Ketamine is similar to fentanyl in that it can act as both an analgesic and an anesthetic, $^{35,36}$ which suggests that these molecules may have independent binding sites, compared to those of general anesthetics, in the context of the GLIC structure.

In conclusion, we have presented a novel binding site for the opioid analgesic/anesthetic fentanyl, which, to the best of our knowledge, is the first published evidence of fentanyl 
interacting with and modulating the conductance state of GLIC. Our simulations have shown that fentanyl binding induces the closure of the helix pore by causing helix rotation and curvature with minimal tilting, leading to the 233-Ile and 240-Ile residues forming a hydrophobic gate blocking the pore conductance, which is behavior that is similar to that identified for general anesthetics. However, with the use of the TRAJELIX module, ${ }^{25}$ we were able to identify a rotational motion of the helices that is not observed for anesthetic binding. Discovery of this modulation of GLIC by fentanyl should stimulate further investigations at an atomic level into the role of opioid analgesics in general anesthesia to provide a more complete description of the mechanisms of general anesthesia.

\section{ASSOCIATED CONTENT}

\section{(5) Supporting Information}

The Supporting Information is available free of charge on the ACS Publications website at DOI: 10.1021/acs.biochem.9b00881.

Methodology, Tables S1-S9, and Figures S1-S10 (PDF)

\section{AUTHOR INFORMATION}

\section{Corresponding Authors}

*Email: FaulknerC3@cardiff.ac.uk.

*Email: deLeeuwN@cardiff.ac.uk.

\section{ORCID $\odot$}

Christopher Faulkner: 0000-0002-5142-8109

Nora H. de Leeuw: 0000-0002-8271-0545

\section{Author Contributions}

C.F., D.F.P., and N.H.d.L. designed the research. C.F. carried out the simulations and analysis. C.F. and N.H.d.L. wrote the manuscript.

\section{Funding}

The authors acknowledge the Engineering and Physical Sciences Research Council (Grant EP/R512503/1) and AWE for funding.

\section{Notes}

The authors declare no competing financial interest.

\section{ACKNOWLEDGMENTS}

This research was undertaken using the Supercomputing Facilities at Cardiff University operated by ARCCA on behalf of CSF and Supercomputing Wales. This equipment is partially funded by the Welsh European Funding Office (WEFO) as well as by the University. Information about the data underpinning the results presented here, including how to access them, can be found in the Cardiff University data catalogue at http://doi.org/10.17035/d.2019.0080928584.

\section{ABBREVIATIONS}

GLIC, Gloeobacter violaceus ion channel; MD, molecular dynamics; TMD, transmembrane domain; MMPSA, molecular mechanics Poisson-Boltzmann surface area; RMSD, rootmean-square deviation; DOPC, dioleoyl-sn-glycero-3-phosphocholine.

\section{REFERENCES}

(1) Mi, W. D., Sakai, T., Singh, H., Kudo, T., Kudo, M., and Matsuki, A. (1999) Hypnotic endpoints vs. the bispectral index,95\% spectral edge frequency and median frequency during propofol infusion with or without fentanyl. Eur. J. Anaesthesiol. 16, 47-52.

(2) Wynands, J. E., Townsend, G. E., Wong, P., Whalley, D. G., Srikant, C. B., and Patel, Y. C. (1983) Blood Pressure Response and Plasma Fentanyl Concentrations during High-and Very High-Dose Fentanyl Anesthesia for Coronary Artery Surgery. Anesth. Analg. 62, 661-665.

(3) Unwin, N. (1993) Neurotransmitter action: Opening of ligandgated ion channels. Cell 72, 31-41.

(4) Campagna, J. A., Miller, K. W., and Forman, S. A. (2003) Mechanisms of Actions of Inhaled Anesthetics. N. Engl. J. Med. 348, $2110-2124$.

(5) Krasowski, M. D., and Harrison, N. L. (1999) General anaesthetic actions on ligand-gated ion channels. Cell. Mol. Life Sci. $55,1278-1303$.

(6) Sauguet, L., Poitevin, F., Murail, S., Van Renterghem, C., Moraga-Cid, G., Malherbe, L., Thompson, A. W., Koehl, P., Corringer, P. J., Baaden, M., and Delarue, M. (2013) Structural basis for ion permeation mechanism in pentameric ligand-gated ion channels. EMBO J. 32, 728-741.

(7) Arcario, M. J., Mayne, C. G., and Tajkhorshid, E. (2017) A membrane-embedded pathway delivers general anesthetics to two interacting binding sites in the Gloeobacter violaceus ion channel. J. Biol. Chem. 292, 9480-9492.

(8) Nury, H., Van Renterghem, C., Weng, Y., Tran, A., Baaden, M., Dufresne, V., Changeux, J. P., Sonner, J. M., Delarue, M., and Corringer, P. J. (2011) X-ray structures of general anaesthetics bound to a pentameric ligand-gated ion channel. Nature 469, 428-431.

(9) Pan, J., Chen, Q., Willenbring, D., Mowrey, D., Kong, X.-P., Cohen, A., Divito, C. B., Xu, Y., and Tang, P. (2012) Structure of the pentameric ligand-gated ion channel GLIC bound with anesthetic ketamine. Structure 20, 1463-1469.

(10) Willenbring, D., Liu, L. T., Mowrey, D., Xu, Y., and Tang, P. (2011) Isoflurane Alters the Structure and Dynamics of GLIC. Biophys. J. 101, 1905-1912.

(11) Chiara, D. C., Gill, J. F., Chen, Q., Tillman, T., Dailey, W. P., Eckenhoff, R. G., Xu, Y., Tang, P., and Cohen, J. B. (2014) Photoaffinity Labeling the Propofol Binding Site in GLIC. Biochemistry 53, 135-142.

(12) Cheng, M. H., Coalson, R. D., and Tang, P. (2010) Molecular Dynamics and Brownian Dynamics Investigation of Ion Permeation and Anesthetic Halothane Effects on a Proton-Gated Ion Channel. J. Am. Chem. Soc. 132, 16442-16449.

(13) Brannigan, G., LeBard, D. N., Henin, J., Eckenhoff, R. G., and Klein, M. L. (2010) Multiple binding sites for the general anesthetic isoflurane identified in the nicotinic acetylcholine receptor transmembrane domain. Proc. Natl. Acad. Sci. U. S. A. 107, 14122-14127.

(14) LeBard, D. N., Hénin, J., Eckenhoff, R. G., Klein, M. L., and Brannigan, G. (2012) General anesthetics predicted to block the GLIC pore with micromolar affinity. PLoS Comput. Biol. 8, No. e1002532.

(15) Mowrey, D., Cheng, M. H., Liu, L. T., Willenbring, D., Lu, X., Wymore, T., Xu, Y., and Tang, P. (2013) Asymmetric ligand binding facilitates conformational transitions in pentameric ligand-gated ion channels. J. Am. Chem. Soc. 135, 2172-2180.

(16) Miller, B. R., McGee, T. D., Swails, J. M., Homeyer, N., Gohlke, H., and Roitberg, A. E. (2012) MMPBSA.py: An efficient program for end-state free energy calculations. J. Chem. Theory Comput. 8, 33143321.

(17) Michaud-Agrawal, N., Denning, E. J., Woolf, T. B., and Beckstein, O. (2011) MDAnalysis: A toolkit for the analysis of molecular dynamics simulations. J. Comput. Chem. 32, 2319-2327.

(18) Jia, Z., Yazdani, M., Zhang, G., Cui, J., and Chen, J. (2018) Hydrophobic gating in BK channels. Nat. Commun. 9, 3408.

(19) Humphrey, W., Dalke, A., and Schulten, K. (1996) VMD: Visual molecular dynamics. J. Mol. Graphics 14, 33-38.

(20) Zhu, F., and Hummer, G. (2010) Pore opening and closing of a pentameric ligand-gated ion channel. Proc. Natl. Acad. Sci. U. S. A. 107, 19814-19819. 
(21) daCosta, C. J. B., and Baenziger, J. E. (2013) Gating of Pentameric Ligand-Gated Ion Channels: Structural Insights and Ambiguities. Structure 21, 1271-1283.

(22) Miyazawa, A., Fujiyoshi, Y., and Unwin, N. (2003) Structure and gating mechanism of the acetylcholine receptor pore. Nature 423, 949-955.

(23) Smart, O. S., Neduvelil, J. G., Wang, X., Wallace, B. A., and Sansom, M. S. P. (1996) HOLE: A program for the analysis of the pore dimensions of ion channel structural models. J. Mol. Graphics 14, 354-360.

(24) Zhu, F., and Hummer, G. (2012) Drying Transition in the Hydrophobic Gate of the GLIC Channel Blocks Ion Conduction. Biophys. J. 103, 219-227.

(25) Mezei, M., and Filizola, M. (2006) TRAJELIX: A Computational Tool for the Geometric Characterization of Protein Helices During Molecular Dynamics Simulations. J. Comput.-Aided Mol. Des. 20, 97-107.

(26) Mezei, M. (2010) Simulaid: A simulation facilitator and analysis program. J. Comput. Chem. 31, 2658-2668.

(27) Dahl, A. C. E., Chavent, M., and Sansom, M. S. P. (2012) Bendix: intuitive helix geometry analysis and abstraction. Bioinformatics 28, 2193-2194.

(28) Pettersen, E. F., Goddard, T. D., Huang, C. C., Couch, G. S., Greenblatt, D. M., Meng, E. C., and Ferrin, T. E. (2004) UCSF Chimera-A visualization system for exploratory research and analysis. J. Comput. Chem. 25, 1605-1612.

(29) Law, R. J., and Lightstone, F. C. (2009) Modeling neuronal nicotinic and GABA receptors: Important interface salt-links and protein dynamics. Biophys. J. 97, 1586-1594.

(30) Venkatachalan, S. P., and Czajkowski, C. (2008) A conserved salt bridge critical for GABAA receptor function and loop C dynamics. Proc. Natl. Acad. Sci. U. S. A. 105, 13604-13609.

(31) Taly, A., Hénin, J., Changeux, J. P., and Cecchini, M. (2014) Allosteric regulation of pentameric ligand-gated: An emerging mechanistic perspective ion channels. Channels 8,350 .

(32) Spurny, R., Billen, B., Howard, R. J., Brams, M., Debaveye, S., Price, K. L., Weston, D. A., Strelkov, S. V., Tytgat, J., Bertrand, S., Bertrand, D., Lummis, S. C. R., and Ulens, C. (2013) Multisite Binding of a General Anesthetic to the Prokaryotic Pentameric Erwinia chrysanthemi Ligand-gated Ion Channel (ELIC). J. Biol. Chem. 288, 8355-8364.

(33) Fourati, Z., Howard, R. J., Heusser, S. A., Hu, H., Ruza, R. R., Sauguet, L., Lindahl, E., and Delarue, M. (2018) Structural Basis for a Bimodal Allosteric Mechanism of General Anesthetic Modulation in Pentameric Ligand-Gated Ion Channels. Cell Rep. 23, 993-1004.

(34) Bondarenko, V., Mowrey, D., Liu, L. T., Xu, Y., and Tang, P. (2013) NMR resolved multiple anesthetic binding sites in the TM domains of the $\alpha 4 \beta 2 \mathrm{nAChR}$. Biochim. Biophys. Acta, Biomembr. 1828, 398-404.

(35) Tyler, M. W., Yourish, H. B., Ionescu, D. F., and Haggarty, S. J. (2017) Classics in Chemical Neuroscience: Ketamine. ACS Chem. Neurosci. 8, 1122-1134.

(36) Yamakura, T., Chavez-Noriega, L. E., and Harris, R. A. (2000) Subunit-dependent Inhibition of Human Neuronal Nicotinic Acetylcholine Receptors and Other Ligand-gated Ion Channels by Dissociative Anesthetics Ketamine and Dizocilpine. Anesthesiology 92, 1144-1153. 